\title{
ANÁLISE DE SENSIBILIDADE DO ROI: UM ESTUDO DE CASO PARA UMA EMPRESA DE TECNOLOGIA DA INFORMAÇÃO
}

\section{SENSITIVITY ANALYSIS OF ROI: A CASE STUDY FOR AN INFORMATION TECHNOLOGY COMPANY}

0 artigo foi aprovado e apresentado no II Congresso UFU de Contabilidade com o título: "Análise de Sensibilidade do ROI: um estudo aplicado", realizado de 19/10/2017 a 20/07/2017, em Uberlândia (MG)

\section{RESUMO}

É sabido que a informação contábil apresenta um problema quanto a sua temporariedade. No caso, ela é uma informação fixa no tempo, baseada em acontecimentos passados. Neste sentido, técnicas estatísticas auxiliam na compreensão da informação para planejamento e atos futuros. O presente trabalho tem por objetivo analisar o comportamento das variáveis que compõem o ROI através da análise de sensibilidade. $\mathrm{O}$ estudo desenvolveu-se como uma pesquisa empírica na qual foram analisados os balancetes mensais do ano de 2015 de uma empresa de tecnologia da cidade de Ribeirão Preto. Para a determinação do output foram utilizados inputs como: ativo circulante e permanente, passivo operacional, receitas, custos, despesas administrativas e comerciais, número de clientes, além de outras despesas cujo montante verificou-se relevante. Por fim, após submeter os inputs ao teste de sensibilidade e simulação de Monte Carlo, foi observado que as variáveis relacionadas a custos e despesas apresentaram correlação negativa próxima de -1 , mostrando, assim, seu impacto negativo no ROI. Já as variáveis como vendas e número de clientes tiveram correlação positiva próxima a +1 , portanto com impacto positivo em relação ao ROI.

Palavras-chave: Análise de Sensibilidade; Retorno sobre o Investimento; Contabilidade Gerencial; Processo de Tomada de Decisão.

\begin{abstract}
It is known that the accounting information presents a problem of temporariness. In this case, it is time-bound information based on past events. In this sense, statistical techniques help in the understanding of the information for planning and future actions. The present work has the objective of analyzing the behavior of the variables that make up the ROI through a sensitivity analysis. The study was developed as an empirical research in which the monthly balance sheets of an information technology company of Ribeirão Preto for the year 2015 were analyzed. In order to determine the output, we used inputs such as: current and permanent assets, operating liabilities, revenues, costs, administrative and commercial expenses, number of customers, and other expenses whose amount was significant. Finally, after subjecting the inputs to the Monte Carlo simulation and sensitivity test, it was observed that the variables related to costs and expenses presented negative correlation close to -1 , thus showing its negative impact on ROI. On the other hand, the variables such as sales and number of customers had a positive correlation close to +1 , with a positive impact on $R O I$.
\end{abstract}

Keywords: Sensitivity Analysis; Return on Investment; Managerial Accounting; Decision Making Process.
Bruno Rodrigues Ferreira Xavier Graduado em Ciências Contábeis pela Universidade de São Paulo (USP) na

Faculdade de Economia, Administração e Contabilidade de Ribeirão Preto (FEARP) Contato: Avenida Bandeirantes, 3900 Monte Alegre, Ribeirão Preto, SP, CEP: 14.040-905. E-mail: brfxavier@gmail.com

Roni Cleber Bonizio

Doutor e Mestre em Controladoria e Contabilidade pela Universidade de São Paulo (USP) na Faculdade de Economia, Administração e Contabilidade de Ribeirão Preto (FEARP). Graduado em Ciências Contábeis pela Universidade de São Paulo (USP) na Faculdade de Economia, Administração e Contabilidade de Ribeirão Preto (FEARP). Docente do departamento de Ciências Contábeis na Universidade de São Paulo (USP) na Faculdade de Economia, Administração e Contabilidade de Ribeirão Preto (FEARP). Contato: Avenida Bandeirantes, 3900, Monte Alegre, Ribeirão Preto, SP, CEP: 14.040-905. E-mail: rbonizio@usp.br 


\section{INTRODUÇÃO}

Em um ambiente altamente competitivo e com recursos escassos, o gerenciamento das organizações torna-se um imperativo. A forma com que estas organizações alocam seus recursos é determinante para a continuidade do negócio (WILLIAMSON, 1991).

É neste sentido que a contabilidade pode contribuir para a continuidade da organização. De um lado temos a contabilidade financeira, cujo objetivo é a geração de informações financeiras para que os usuários externos possam fazer avaliações do desempenho financeiro da companhia. Do outro lado a contabilidade gerencial, que possui foco direcionado para o usuário interno e serve de base para a gestão da empresa. A atuação da contabilidade gerencial ocorre por meio dos relatórios gerenciais, que são utilizados como base para a tomada de decisão (SCOTT, 2015).

De fato, da mesma forma que estes relatórios são usados no processo de gestão, eles possuem algumas limitações relevantes. Tais relatórios trabalham com informações de eventos passados, sem aproveitar a contribuição que o histórico daquelas informações pode gerar.

Desta forma, para este trabalho, foi selecionado o Retorno sobre Investimento (ROI) como variável de interesse (output). O ROI é um importante indicador de desempenho operacional, pois mede o nível de retorno que o investimento está gerando. De acordo com Assaf Neto (2014), este indicador reflete a relação entre o lucro operacional gerado e os ativos deduzidos dos passivos de funcionamento.

Para o desenvolvimento do trabalho, foram utilizados balancetes mensais do ano de 2015 de uma empresa de tecnologia de Ribeirão Preto, estado de São Paulo ${ }^{1}$. A empresa objeto tem médio porte, com faturamento anual próximo a 40 milhões de reais, atuando há mais de 25 anos no mercado em que está inserida.

Segmentando os dados financeiros da empresa, e acrescentando um indicador não-financeiro (o número de clientes), este trabalho buscou responder a seguinte pergunta: Dentre os inputs selecionados, qual deles tem maior sensibilidade e relevância para a geração do output ROI?

A contribuição deste trabalho será também em estimar a correlação destes inputs fornecendo uma base confiável de mensuração destes valores. A correlação dos inputs com os outputs tem a capacidade de indicar ao gestor quais variáveis de interesse são mais significativas na tomada de decisão.

Este trabalho está dividido em mais quatro partes. A segunda parte reúne a fundamentação teórica sob a qual o trabalho foi desenvolvido. Na terceira parte, há a abordagem de toda a metodologia dos testes que foram realizados. A seguir, na quarta parte, resultados obtidos são discutidos. Por fim, a última parte é composta por uma conclusão, abordando aspectos mais ligados aos objetivos e limitações do trabalho.

\section{REFERENCIAL TEÓRICO}

Esta parte apresenta-se como um suporte teórico para as questões que são discutidas neste trabalho. Ele é composto por três seções, sendo a primeira uma descrição do indicador chave para o trabalho, o ROI. A segunda discorre sobre a Análise de Sensibilidade, um processo utilizado para a avaliação do ROI. Já o terceiro item desta parte apresenta processos de simulação de cenários futuros envolvendo o indicador e seus principais componentes.

\subsection{Retorno sobre o Investimento (ROI)}

O Retorno sobre o Investimento (ROI) é um indicador muito popular na análise de investimentos, sendo usado para medir o retorno financeiro passado ou estimar tais retornos no futuro (ASSAF NETO, 2014). De forma clara, este indicador apresenta uma taxa de retorno, estimando o quanto o investidor obteve de retorno em relação ao valor aplicado em um investimento específico. Para tanto, o ROI procura relacionar o lucro operacional com o investimento realizado.

O cálculo do ROI é apresentado na equação (1) e busca evidenciar as variáveis que determinam o desempenho operacional da organização.

$$
R O I=M O_{l i q} \times \text { Giro }_{\text {inv }}
$$

Sendo $\mathrm{MO}_{\text {liq }}$ a margem operacional líquida e Giro ${ }_{\text {inv }}$ o giro do investimento.

Para uma melhor compreensão do que representa a Margem Operacional Líquida na composição do ROI, Assaf Neto (2014) afirma que:

A Margem Operacional demonstra o desempenho da empresa medido em função de valores efetivamente utilizados em suas operações normais. Quanto das receitas de vendas foi destinado a cobrir despesas operacionais, e quanto se transformou efetivamente em lucro.

Portanto, o cálculo da Margem Operacional Líquida permite que seja feita uma análise do comportamento das receitas, despesas e custos incorridos pela organização. A fórmula para encontrar a margem operacional líquida de uma empresa está em (2).

Por motivos de confidencialidade dos dados, o nome da empresa foi mantido em sigilo. 


$$
M O_{\text {liq }}=\text { Lucro }_{o p} / \text { Receitas }_{o p}
$$

Com Lucro op o lucro operacional e Receitas ${ }_{\text {op }}$ as receitas totais operacionais.

Por sua vez, o Giro do Investimento reflete a quantidade de vezes que o ativo total da empresa 'girou' em um período específico em função das vendas realizadas. Logo, quanto maior for o 'giro’ obtido, melhor terá sido o desempenho da companhia (ASSAF NETO, 2014). O cálculo do giro do investimento é apresentado em (3).

$$
\text { Giro }_{\text {inv }}=\text { Receitas }_{\text {op }} \times \text { Investimento }
$$

É possível, então, resumir o ROI como um indicador cuja influência de dois fatores operacionais $\left(\mathrm{MO}_{\text {liq }}\right.$ e Giro $\left.{ }_{\text {inv }}\right)$ demonstra a agregação de utilidade aos produtos e serviços da empresa e também a sua eficiência na aplicação do capital investido (BONIZIO, 2005).

Ball e Brown (1968) foram pioneiros na busca da relação entre os ganhos contábeis sobre os preços das ações. Jacobson (1987) buscou complementar este tema ao analisar a validação do ROI como medida de performance organizacional, e também o impacto dessa medida sobre o preço das ações de 241 companhias norte-americanas entre os anos de 1963 e 1982. O autor analisou e encontrou correlação linear estatisticamente significante que corrobora com a hipótese de eficiência de mercado. Ainda, o trabalho ressalta a importância deste indicador como medida de desempenho econômico das empresas.

Neste sentido, a literatura do ROI se desenvolveu na busca de analisá-lo como indicador de desempenho das organizações. Recentemente, Ichsani e Suhardi (2015) analisaram a relação entre o ROI e o ROE com o volume de ações das companhias de capital aberto da Indonésia para os anos de 2009 a 2013. Os resultados das estimações demonstraram que ambos indicadores de rentabilidade impactam o volume das ações. Kang, Khaksari e Nam (2018) também encontraram que a rentabilidade da companhia é um importante direcionador de liquidez no mercado.

Recentemente, o ROI é estudado também na literatura de pesquisa operacional, a qual utiliza o indicador como forma de parâmetro inicial para firmas planejarem uma alocação ótima de seus recursos. Menezes, Kim e Huang (2015) desenvolveram um modelo de alocação ótima com o objetivo principal de maximizar o lucro sujeito a um ROI mínimo requerido para cada loja de uma empresa. Dentre os inputs utilizado estavam: localização, número de lojas, projeção de demanda e investimento de cada loja.

Kristjansdottir et al. (2018) analisaram o papel dos sistemas operacionais de produção das companhias sobre a redução do consumo de recursos produtivos, dentre eles a redução do tempo de fabricação, aumento da qualidade dos produtos e aumento das vendas. Em suma, os autores analisaram, por meio de um estudo de caso em uma empresa multinacional de produção de bombas, como que o sistema operacional de produção, que fornece suporte desde o processo produtivo até o processo da venda, pode afetar positivamente o retorno sobre os investimentos (ROI) realizado pela companhia.

Dessa forma, pode ser observado que os trabalhos que compõem a literatura sobre o ROI ainda não buscaram analisar quais variáveis contribuem para o aumento deste indicador de desempenho por meio de uma análise de sensibilidade via simulação de Monte Carlo. Assim, esta importante análise compõe o objetivo do presente trabalho, visto que, dada a restrição temporal, é importante que os gestores saibam qual variável organizacional devem alterar para que se aumente a rentabilidade e desempenho das companhias.

\subsection{Análise de sensibilidade}

O modelo de análise de sensibilidade utilizado neste trabalho tem como base Bonizio (2005). A análise de sensibilidade é uma ferramenta útil no processo de decisão da empresa, pois consegue mensurar a relação que os inputs têm com o output estudado (SALTELLI, 2002).

Dentre as diversas aplicações da análise de sensibilidade, a tomada de decisão pode ser considerada a principal. Para Samson (1988), a "análise de sensibilidade é uma importante etapa da decisão". Isto ocorre, pois a análise de sensibilidade fornece aos gestores a possibilidade de compilar diversas informações em um único output, além de testá-los em cenários futuros.

O Quadro 1 auxilia na compreensão da análise de sensibilidade como instrumento no processo de tomada de decisão ao apresentar as fases para decisão. 
Quadro 1 - Questões da análise de sensibilidade para tomada de decisão.

\begin{tabular}{|c|c|}
\hline Fases da Decisão & Questões da Análise de Sensibilidade \\
\hline Definição do problema real & $\begin{array}{l}\text { Qual é o problema real e como as suas várias definições afetam nosso } \\
\text { comportamento na decisão? }\end{array}$ \\
\hline Construção do modelo & $\begin{array}{l}\text { Quais as possíveis abordagens e estruturas alternativas na modelagem? } \\
\text { Qual é o efeito de mudanças nos valores assumidos dos parâmetros? }\end{array}$ \\
\hline Solução do modelo & Qual é o efeito das mudanças no critério de decisão? \\
\hline Interpretação do modelo & $\begin{array}{l}\text { Como o processo de modelagem muda ou deve mudar nosso pensamento } \\
\text { sobre o problema? }\end{array}$ \\
\hline Escolha final & $\begin{array}{l}\text { Qual é a relevância da última linha de decisão (modelo produzido) para o } \\
\text { problema real? }\end{array}$ \\
\hline Ação real & Quão bem a ação real resolve o problema real? \\
\hline
\end{tabular}

Fonte: Samson, 1988, p.272.

Na definição do problema real, o gestor tende a deparar-se com o excesso de informações geradas por diversos relatórios e sistemas de controles. Por vezes, tais informações podem ser confusas e atrapalham a tomada de decisão. Neste sentido, a análise de sensibilidade pode contribuir ao medir a relevância destas informações (SALTELLI, 2002; SAMSON, 1988).

Para a construção do melhor modelo a ser utilizado na tomada da decisão, o gestor precisa levar em consideração quais informações devem ser incluídas, bem como a forma como estas informações serão abordadas e a contribuição delas para a decisão. Desta maneira, a análise de sensibilidade pode exemplificar a abordagem de diferentes modelos. Na prática, esta comparação entre modelos é possível através da estimação do grau de relacionamento entre as variáveis envolvidas, possibilitando ao gestor encontrar o melhor arranjo entre as informações disponíveis (SALTELLI, 2002; SAMSON, 1988).

Para a fase de solução do modelo, o gestor deve selecionar as regras de decisão, tendo em vista que os diversos elementos envolvidos têm impacto no critério de decisão e nos resultados a serem obtidos. Logo, a análise de sensibilidade pode estimar o comportamento destes elementos e auxiliar na determinação das regras de decisão (SALTELLI, 2002; SAMSON, 1988).

Para a fase de interpretação, a análise de sensibilidade pode contribuir para uma revisão do modelo adotado, permitindo uma reavaliação dos critérios utilizados (SALTELLI, 2002; SAMSON, 1988).

Outra contribuição da análise de sensibilidade é no momento final para a tomada de decisão, no qual o gestor pode decidir contemplar variáveis que antes não compunham o modelo inicial. É, então, possível medir a relevância destas novas variáveis, ou seja, como elas se comportam no modelo afetando, assim, o output (SALTELLI, 2002; SAMSON, 1988).

Por fim, a Análise de Sensibilidade pode tornar o modelo mais completo, tendo impacto positivo na tomada de decisão. Pode, ainda, permitir a estimação de cenários futuros, organizando melhor os inputs para que os outputs possam ser mais relevantes (SAMSON, 1988).

Cabe ressaltar que não houve trabalhos na literatura que utilizassem o método de simulação de Monte Carlo para analisar a sensibilidade do ROI. Sendo assim, a Análise de Sensibilidade fornece ao gestor outputs mais precisos, permitindo um melhor conhecimento da situação analisada, e, consequentemente, melhorando a decisão a ser tomada. É importante colocar ainda, que a decisão estará mais bem sustentada se passar por todas as fases descritas nos quadros acima.

\section{METODOLOGIA}

Esta parte apresenta a forma como a pesquisa foi desenvolvida. Desta forma, o presente estudo caracteriza-se como um estudo de caso. Esta estratégia de pesquisa consiste no estudo em profundidade de um ou poucos objetos, permitindo, assim, seu amplo e detalhado conhecimento (GIL, 2002). A primeira subseção apresenta a empresa foco deste estudo e como foi montado o banco de dados. A segunda subseção apresenta a decomposição do ROI. Por fim, a terceira subseção demonstra os passos para a realização da simulação de Monte Carlo.

\subsection{Descrição da empresa e montagem do banco de dados}

A empresa escolhida tem sede na cidade de Ribeirão Preto, estado de São Paulo, e atua no ramo de desenvolvimento, produção e comercialização de soluções em tecnologia da informação para setores de distribuição, atacado e varejo. Sua área de atuação é abrangente, com clientes em todo o país. Seu faturamento anual é de aproximadamente $\mathrm{R} \$ 40$ milhões e tem por volta de 300 colaboradores. O banco de dados desta pesquisa é composto pelos balancetes mensais consolidados de 2015 da empresa selecionada. 
Após a organização dos balancetes em um único banco de dados, foi realizada a segmentação das contas contábeis por grupos. Vale ressaltar que estes grupos serão os inputs neste processo de modelagem para a geração do ROI.

Para as contas patrimoniais, foram selecionadas apenas as contas que estão diretamente relacionadas à operação da empresa. Foram excluídas desta segmentação contas que não pertencem à operação da companhia, tais como contas de investimentos específicos e financiamentos para outros fins, além das contas do Patrimônio Líquido. O Quadro 2 descreve esta segmentação.

Quadro 2 - Segmentação das contas patrimoniais.

\begin{tabular}{|c|c|c|}
\hline Ativo & CAIXA & \\
\hline Ativo & BANCOS & \\
\hline Ativo & APLICAÇÕES FINANCEIRAS - CP & \\
\hline Ativo & OUTROS CRÉDITOS - CP & CIRCULANTE \\
\hline Ativo & CRÉDITOS TRIBUTÁRIOS - CP & \\
\hline Ativo & ESTOQUES & \\
\hline Ativo & CONTAS A RECEBER - LP & \\
\hline Ativo & BENS EM OPERAÇÃO - LP & \\
\hline Ativo & BENS EM OPERAÇÃO - DEPRECIAÇÃO & PERMANENTE \\
\hline Ativo & BEM INTANGÍVEL - LP & \\
\hline Passivo & EMPRÉSTIMOS E FINANCIAMENTOS MOEDA - CP & \\
\hline Passivo & FORNECEDORES DIVERSOS - CP & \\
\hline Passivo & OBRIGAÇÕES TRABALHISTAS - CP & $\begin{array}{l}\text { PASSIVO FUNCIONAL / } \\
\text { OPERAÇÃO }\end{array}$ \\
\hline Passivo & PROVISÕES PARA FÉRIAS E $13^{\circ}$. SALA - CP & \\
\hline Passivo & OBRIGAÇÕES TRIBUTÁRIAS - CP & \\
\hline
\end{tabular}

Fonte: Elaboração própria.

Para as contas de resultado, foi realizada uma segmentação abrangendo os grupos de contas cuja movimentação pertence à operação da empresa. Logo, as contas de resultados financeiros e outras receitas e despesas não operacionais não foram consideradas. O Quadro 3 sintetiza a segmentação.

Quadro 3 - Segmentação das contas de resultado.

\begin{tabular}{|l|c|}
\hline Resultado & VENDAS / FATURAMENTO \\
Resultado & CUSTOS \\
Resultado & DESPESAS COMERCIAIS \\
Resultado & DESPESAS ADMINISTRATIVAS \\
Resultado & OUTRAS DESPESAS \\
\hline
\end{tabular}

Fonte: Elaboração própria.

Outra variável que será utilizada neste modelo e não está contemplada no plano de contas é o número de clientes, variável coletada junto à própria empresa e de caráter não financeiro. Esta medida será utilizada, pois é de mensuração confiável e está claramente relacionada com as variáveis Vendas/Faturamento, Custos e Despesas Comerciais. 


\subsection{Modelo para apuração do ROI}

A Figura 1 evidencia a forma como foi modelada a geração do output ROI através dos inputs mencionados.

Figura 1 - Decomposição do ROI.

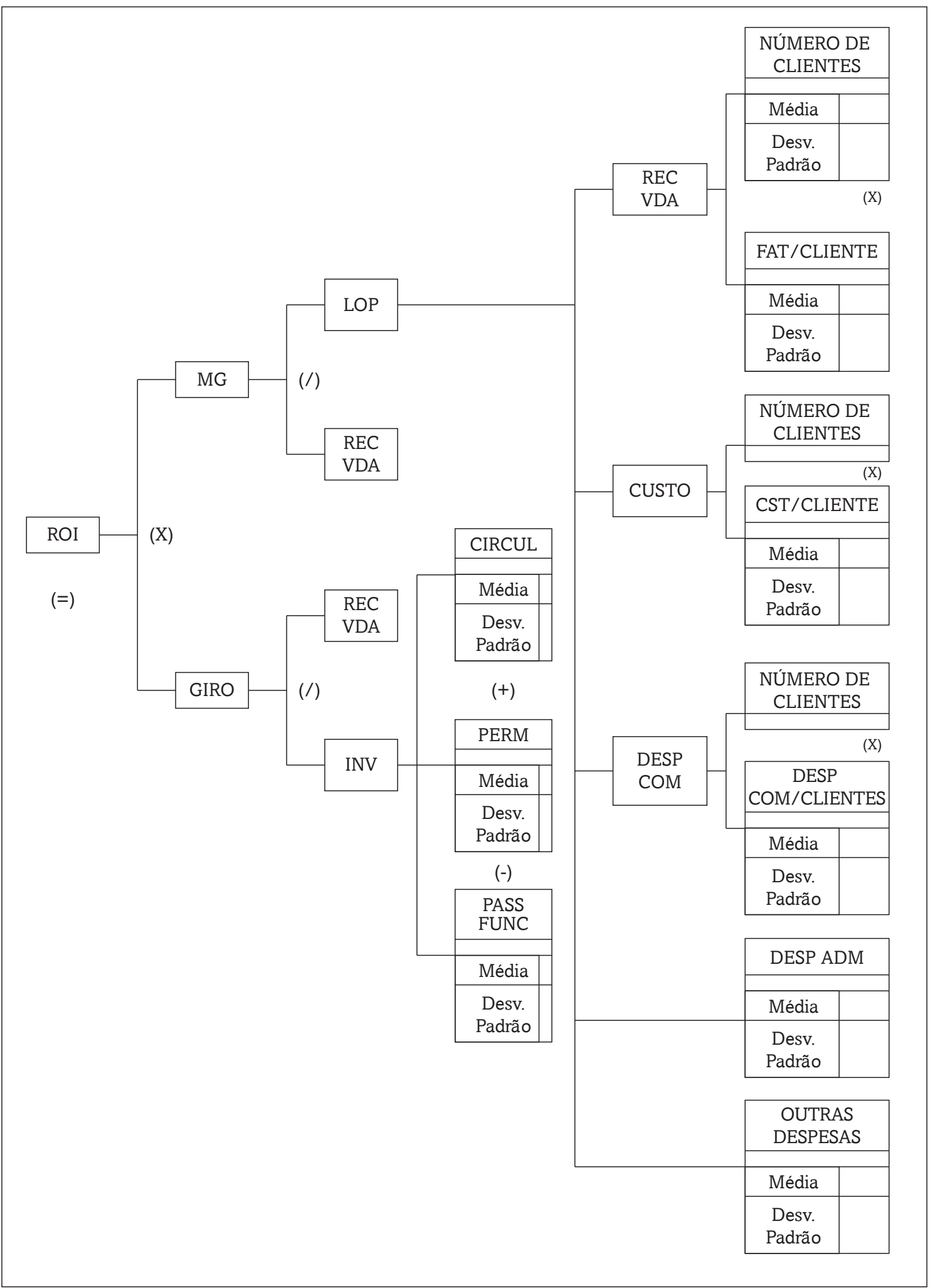

Fonte: Elaboração própria. 
É importante observar que, apesar dos inputs refletirem aspecto particular da empresa estudada, os outros itens (Lucro Op., Vendas, Invest., Margem, Giro e ROI) estão de acordo com o modelo sugerido por Assaf Neto (2014).

Os valores classificados como custo, despesa comercial e despesa administrativa seguem uma divisão da própria empresa utilizando contas com a mesma nomenclatura para cada um desses grupos. Por exemplo, o grupo de contas de pessoal (salários, férias, $13^{\circ}$ salário, FGTS, INSS e todas as outras contas decorrentes de verbas com pessoal) se repete nos três grupos. Dessa forma, a empresa apropria as verbas de pessoal como custo para aqueles colaboradores que trabalham na produção e desenvolvimento do software. Como despesa comercial, são alocados os valores de pessoal das equipes de vendas e pós-vendas. Para as despesas administrativas, são alocadas as despesas provenientes dos departamentos de apoio (administrativo, financeiro, recursos humanos e outros).

Para o grupo de contas classificado como outras despesas, são alocados os valores contabilizados como receitas e despesas não operacionais, tais como resultado com imobilizado, perdas com clientes, outras receitas, reversão de provisões. É preciso lembrar que tais classificações para contabilização são feitas pela própria empresa.

O input REC VDA (Receita de Vendas) foi obtido através da relação entre o faturamento da empresa e a quantidade de clientes atendidos (dados fornecidos pela empresa). Dessa forma, estabelecemos o LOP (Lucro Operacional) como a receita de vendas menos o custo, as despesas comerciais, despesas administrativas e outras despesas, e a MG (Margem Operacional Líquida) como sendo o LOP dividido pela REC VDA.

Do outro lado da equação, o GIRO é obtido a partir da divisão da REC VDA pelo Investimento. E o investimento é o resultado do grupo de inputs circulante (CIRCUL), mais o ativo permanente (PERM), menos o passivo de funcionamento (PASS FUNC).

Neste modelo de apuração do ROI foram executadas as seguintes etapas: (1) levantamento dos dados contábeis da empresa selecionada; (2) segmentação das contas contábeis em grupos específicos (inputs); (3) apuração da média trimestral e respectivos desvios padrão de cada input; (4) os valores considerados inputs foram inseridos na tabela de apuração do ROI; e (5) realização do processo de simulação a partir dos inputs obtidos

A partir do processo de simulação, foi possível a obtenção de uma amostra com 100.000 valores estimados para cada componente do ROI. Então, por esta amostra, foi possível estabelecer o coeficiente de correlação de cada input em relação ao output selecionado.

Na Tabela 1 é apresentada uma estatística descritiva dos inputs apurados após a análise dos balancetes.

Tabela 1 - Estatística descritiva das variáveis.

\begin{tabular}{c|l|c|c|c}
\hline Tipo & Grupo & Média (em mil R\$) & $\begin{array}{c}\text { Desvio Padrão } \\
\text { (em mil R\$) }\end{array}$ & Variação \\
\hline Ativo & CIRCULANTE & $13.316,31$ & 710,22 & $5,33 \%$ \\
Ativo & PERM & $12.841,81$ & $1.459,78$ & $11,37 \%$ \\
Passivo & PASSIVO FUNC & $8.702,90$ & $1.144,63$ & $13,15 \%$ \\
Resultado & VENDAS / FATURAMENTO & $31.012,49$ & 917,27 & $2,96 \%$ \\
Resultado & CUSTOS & $15.662,14$ & $1.294,01$ & $8,26 \%$ \\
Resultado & DESPESAS COMERCIAIS & $1.888,87$ & 71,88 & $3,81 \%$ \\
Resultado & DESPESAS ADMINISTRATIVAS & $1.968,21$ & 304,14 & $15,45 \%$ \\
Resultado & OUTRAS DESPESAS & 408,75 & 66,42 & $16,25 \%$ \\
\hline
\end{tabular}

Elaboração própria.

Para o processo de simulação, foi utilizado o software @Risk, parte de um pacote de soluções chamado Decisions Tools da empresa Palisade. Este software é executado juntamente com uma planilha Microsoft Excel. Com a utilização do @Risk, foi possível estabelecer o comportamento dos inputs para a geração do ROI, bem como a relevância e o impacto de cada um deles numa distribuição probabilística.

\subsection{Simulação de Monte Carlo}

Este estudo usou a Simulação de Monte Carlo, na qual, a partir de um modelo pronto, são geradas amostras aleatórias das variáveis que compõem este modelo. Dessa forma, é possível trabalhar com diversos valores simulados de inputs gerando variados outputs. Este modelo de simulação é útil ao trabalho porque parte de modelos reais, ou seja, relatórios 
gerenciais concretos. Além disso, permite uma amostra de dados aleatória com variabilidade e estimativas para a variável de interesse (GUJARATI, 2005; PHILLIPS; SUL, 2007).

É necessário observar que a ideia de amostras aleatórias deve ser usada aqui com certa cautela, uma vez que tais amostras são geradas a partir de métodos matemáticos pré-definidos. Estas amostras seguem um padrão anteriormente estipulado. Ou seja, ao executar um conjunto de amostras aleatórias cuja média esperada for 'X' e o desvio padrão esperado for 'Y', tais amostras devem refletir este comportamento (GUJARATI, 2005; PHILLIPS; SUL, 2007).

Dessa forma, Watson e Blackstone Junior (1989) afirmam:

[...] para executar a amostragem de Monte Carlo, os números aleatórios devem ser associados com os possíveis valores da variável de modo que a quantidade de números apontados para que cada valor da variável é proporcional a sua probabilidade de ocorrência.

Estabelecendo as regras e gerando as amostras, é possível obter os resultados e analisar o comportamento das variáveis que compõem o modelo. Ou seja, é possível analisar como os inputs se comportam e possíveis cenários simulados gerando diferentes outputs.

Neste sentido, a Simulação de Monte Carlo pode, num primeiro plano, oferecer ao gestor maior qualidade de informação, minimizando o impacto de incertezas acerca do futuro, e num segundo plano, é capaz de medir o nível de sensibilidade dos inputs em diferentes combinações. Tudo isto pode nutrir o gestor com uma informação qualitativamente superior, permitindo que ele identifique e acompanhe quais inputs cuja variação é mais relevante em relação ao output analisado.

\section{ANÁLISE DOS RESULTADOS}

Após a realização do processo de simulação de Monte Carlo, foi possível estabelecer, com maior clareza, o comportamento de cada variável que escolhemos como input para apuração do ROI. Vale lembrar que o objetivo do trabalho não é estabelecer um valor final para o ROI, mas, sim, a sensibilidade dos inputs em relação a ele.

Tem-se então que, tais indicadores tendem a reagir de forma mais ou menos sensível em relação ao output, justificando, assim, sua participação no modelo. Essa reação dos inputs pode ser descrita como o coeficiente de correlação parcial entre as variáveis e o output (GUJARATI, 2005).

O coeficiente de correlação é uma medida estatística de associação linear. A correlação varia de -1 a +1 , onde valores que se aproximam destes números apresenta uma relação linear muito forte, seja negativa para os valores próximos a -1 , ou positiva para os valores próximos a +1 . Já os valores que se aproximam de zero indicam uma correlação fraca, sendo o 0 a ausência de correlação entre as variáveis (GUJARATI, 2005).

A Tabela 2 traz um resumo estatístico para o retorno sobre o investimento.

Tabela 2 - Estatística descritiva para o ROI

\begin{tabular}{c|c}
\hline Estatística & Valor (\%) \\
\hline Mínimo & $-17,63$ \\
Máximo & 24,93 \\
Média & 2,16 \\
Desvio Padrão & 4,20 \\
\hline
\end{tabular}

Fonte: Elaboração própria.

Pela Tabela 2, é possível observar os principais dados estatísticos para o output. No caso, a média para o ROI é de $2,16 \%$ com desvio padrão de $4,20 \%$. No entanto, estes valores podem chegar ao máximo de $24,93 \%$ e ao mínimo de $-17,63 \%$. Tais números refletem uma alta variação no output, e podem proporcionar ao gestor uma visão mais ampla de possíveis cenários a serem enfrentados.

Estes dados são fundamentais para este trabalho, pois cumprem com o objetivo de sair de um modelo determinístico de apuração de indicadores contábeis e passar a admitir a apuração probabilística destes indicadores.

Para continuação da análise, a Tabela 3 apresenta o coeficiente de correlação de cada input na variável de interesse em relação ao ROI.

Tabela 3 - Coeficiente de correlação do ROI

\begin{tabular}{c|c}
\hline Nome & Coeficiente de Correlação \\
\hline CST/CLIENTE & $-0,64$ \\
DESP ADM & $-0,41$ \\
FAT/CLIENTE & 0,41 \\
DESP COM/CLIENTE & $-0,30$
\end{tabular}




\begin{tabular}{c|c}
\hline Nome & Coeficiente de Correlação \\
\hline NÚMERO DE CLIENTES & 0,29 \\
OUTRAS DESPESAS & $-0,90$ \\
INV / PERM & $-0,40$ \\
PASS FUNC & 0,30 \\
CIRCUL & $-0,20$ \\
\hline
\end{tabular}

Fonte: Elaboração própria.

Pela Tabela 3, fica evidente a correlação de cada input com o output. Não obstante, a correlação é uma medida de associação linear e pode não refletir uma causalidade. Uma alta correlação entre duas variáveis não quer dizer que esta alteração em determinada variável causará uma alteração na outra variável. No caso, ambas apenas se comportam em conjunto (GUJARATI, 2005).

É possível observar que as variáveis com maior correlação negativa na geração do ROI são Custo/ $\mathrm{N}^{\circ}$ de clientes com correlação negativa de -0,64, Despesas Administrativas com correlação negativa de -0,41 e Despesas Comerciais/ $\mathrm{N}^{\circ}$ de clientes com correlação negativa de $-0,30$.

Para qualquer gestor, o monitoramento destas três variáveis é fundamental, pois elas possuem uma considerável correlação negativa com o ROI. Essa informação pode ser determinante no processo de tomada de decisão, e deve, inclusive, influenciar na geração de medidas de contenção de gastos e corte de despesas.

Uma saída para o gestor, nesta situação, seria avaliar, entre esse grupo de custos e despesas, quais contas são proporcionalmente relevantes. Por exemplo, sendo a empresa uma prestadora de serviços, convém imaginar que seus maiores gastos estejam em contas de folha de pagamento. Logo, medidas que reduzam os valores gastos com este tipo de despesa podem contribuir para a melhora do ROI.

Por outro lado, algumas variáveis apresentam uma correlação positiva. São elas: Faturamento/ $\mathrm{N}^{\circ}$ de clientes com correlação positiva de 0,41 e $\mathrm{N}^{\circ}$ de clientes com correlação positiva de 0,29.

De fato, estes resultados eram esperados, pois é evidente que indicadores como faturamento e número de clientes contribuam para gerar bons resultados financeiros. Todavia, a contribuição deste modelo é justamente em fornecer um padrão concreto e fidedigno de mensuração destes indicadores. Eles deixam de ser apenas perceptivos para serem conhecidos.

Além do mais, a empresa pode ter sua receita segmentada por grupos de serviços, o que ajudaria na geração de novos faturamentos. A segmentação, nesse caso, pode ser entre os tipos de serviços oferecidos, produtos vendidos aos clientes, customizações em softwares, implantação em novos clientes, contratos de parcerias.

As duas próximas tabelas auxiliam na evidenciação do comportamento dos inputs e como eles afetam a variável de interesse. Estas tabelas mostram alguns resultados obtidos através do processo de Simulação de Monte Carlo.

$\mathrm{Na}$ Tabela 4, foram selecionados os 20 maiores valores para o ROI de acordo com o resultado obtido no processo de Simulação de Monte Carlo.

Tabela 4 - 20 maiores resultados obtidos através da Simulação de Monte Carlo, em mil R\$.

\begin{tabular}{l|c|c|c|c|c|c|c|c|c|c}
\hline $\mathbf{N o m e}$ & $\mathbf{R O I}(\%)$ & $\begin{array}{c}\mathbf{N}^{\mathbf{0}} \text { DE } \\
\text { CLIENTES }\end{array}$ & $\begin{array}{c}\text { FAT/ } \\
\text { CLIENTE }\end{array}$ & $\begin{array}{c}\text { VDAS/CST/ } \\
\text { CLIENTE }\end{array}$ & CIRCUL & $\begin{array}{c}\text { DESP } \\
\text { COM/ } \\
\text { CLIENTE }\end{array}$ & $\begin{array}{c}\text { INV / } \\
\text { PERM }\end{array}$ & $\begin{array}{c}\text { DESP } \\
\text { ADM }\end{array}$ & $\begin{array}{c}\text { PASS } \\
\text { FUNC }\end{array}$ & $\begin{array}{c}\text { OUTRAS } \\
\text { DESP }\end{array}$ \\
\hline $\mathbf{8 2 7 3}$ & 20,40 & 439 & 31.078 & 12.132 & 13.509 & 4.499 & 10.808 & 1.856 & 9.624 & 343 \\
$\mathbf{1 0 2 8 0}$ & 19,56 & 404 & 33.078 & 13.800 & 13.134 & 3.882 & 13.095 & 1.499 & 9.416 & 305 \\
$\mathbf{1 1 5 9 5}$ & 23,31 & 411 & 32.269 & 14.175 & 12.847 & 4.668 & 10.876 & 1.397 & 12.679 & 433 \\
$\mathbf{1 7 4 9 9}$ & 20,12 & 387 & 31.651 & 12.702 & 12.782 & 4.150 & 11.681 & 1.731 & 11.183 & 292 \\
$\mathbf{2 3 0 8 9}$ & 19,68 & 402 & 31.948 & 12.503 & 13.390 & 4.343 & 11.567 & 1.474 & 8.701 & 316 \\
$\mathbf{2 5 0 4 0}$ & 19,76 & 387 & 32.409 & 14.276 & 13.614 & 3.893 & 10.095 & 1.177 & 9.479 & 462 \\
$\mathbf{2 6 5 7 5}$ & 21,72 & 425 & 32.212 & 13.741 & 12.928 & 3.823 & 11.779 & 1.438 & 9.693 & 379 \\
$\mathbf{2 9 4 1 8}$ & 20,40 & 395 & 30.473 & 12.965 & 13.236 & 3.771 & 10.858 & 1.317 & 10.764 & 379 \\
$\mathbf{3 4 5 9 6}$ & 20,65 & 411 & 30.606 & 10.617 & 13.490 & 4.755 & 12.069 & 1.420 & 8.883 & 338 \\
$\mathbf{3 5 5 8 4}$ & 20,18 & 414 & 32.351 & 13.088 & 11.227 & 4.541 & 12.432 & 1.725 & 9.790 & 441 \\
$\mathbf{4 0 0 9 9}$ & 19,94 & 374 & 32.512 & 11.720 & 13.725 & 4.714 & 10.359 & 1.943 & 10.783 & 395
\end{tabular}




\begin{tabular}{l|c|c|c|c|c|c|c|c|c|c}
\hline $\mathbf{N o m e}$ & ROI (\%) & $\begin{array}{c}\mathbf{N}^{\mathbf{0}} \text { DE } \\
\text { CLIENTES }\end{array}$ & $\begin{array}{c}\text { FAT/ } \\
\text { CLIENTE }\end{array}$ & $\begin{array}{c}\text { VDAS/CST/ } \\
\text { CLIENTE }\end{array}$ & CIRCUL & $\begin{array}{c}\text { DESP } \\
\text { COM/ } \\
\text { CLIENTE }\end{array}$ & $\begin{array}{c}\text { INV / } \\
\text { PERM }\end{array}$ & $\begin{array}{c}\text { DESP } \\
\text { ADM }\end{array}$ & $\begin{array}{c}\text { PASS } \\
\text { FUNC }\end{array}$ & $\begin{array}{c}\text { OUTRAS } \\
\text { DESP }\end{array}$ \\
\hline $\mathbf{4 1 8 2 3}$ & 20,11 & 403 & 32.730 & 14.166 & 11.245 & 4.072 & 13.212 & 1.613 & 10.616 & 330 \\
$\mathbf{4 9 2 8 1}$ & 21,70 & 405 & 33.475 & 13.409 & 12.922 & 4.478 & 12.080 & 1.128 & 8.165 & 394 \\
$\mathbf{4 9 6 7 0}$ & 20,00 & 371 & 31.704 & 11.243 & 12.880 & 4.687 & 11.104 & 1.594 & 9.496 & 369 \\
$\mathbf{5 3 1 8 8}$ & 20,97 & 444 & 32.608 & 13.731 & 12.665 & 4.703 & 12.639 & 1.391 & 9.307 & 328 \\
$\mathbf{5 4 4 8 3}$ & 19,74 & 384 & 32.170 & 13.868 & 13.751 & 4.990 & 11.884 & 944 & 11.238 & 286 \\
$\mathbf{6 6 2 4 5}$ & 24,93 & 446 & 32.949 & 10.785 & 12.071 & 4.891 & 11.978 & 1.838 & 7.230 & 430 \\
$\mathbf{7 1 0 2 2}$ & 21,26 & 432 & 31.236 & 13.175 & 12.297 & 4.430 & 11.129 & 1.777 & 11.373 & 414 \\
$\mathbf{7 5 3 4 1}$ & 19,84 & 421 & 32.012 & 12.447 & 14.045 & 4.329 & 11.121 & 1.206 & 6.942 & 462 \\
$\mathbf{8 0 8 5 5}$ & 19,72 & 377 & 31.198 & 12.021 & 12.848 & 5.102 & 9.688 & 1.395 & 9.456 & 343 \\
$\mathbf{9 6 7 7 4}$ & 20,01 & 410 & 32.897 & 12.970 & 12.413 & 3.983 & 12.694 & 1.893 & 9.635 & 420 \\
\hline
\end{tabular}

Fonte: Elaboração própria.

Pela Tabela 4, fica evidente que o aumento do ROI está relacionado à diminuição de variáveis como Custo e Despesas Administrativas. Para o input Custo, foi apurado uma média \$15.662,14 mil de acordo com os balancetes obtidos. Nos resultados acima, esta variável apresenta média de \$12.834,90 mil. Fato semelhante ocorre com a variável Despesas Administrativas onde a apuração dos balancetes apresentou uma média de \$1.968,00 mil. A média desses resultados da simulação ficou em \$1.512,00 mil.

Por outro lado, tais variações substanciais não foram observadas no input Vendas/Faturamento, no qual a média dos balancetes ficou em $\$ 31.012,00$ mil. Já a média dos 20 maiores ROIs obtidos pela simulação ficou em $\$ 32.074,00$ mil. Tal situação corrobora a implementação de políticas de gestão que visam a redução de custos e despesas incorridos.

Na tabela 5, foram selecionados os 20 menores valores para o ROI gerados no processo de Simulação de Monte Carlo.

Tabela 5 - 20 menores resultados obtidos através da Simulação de Monte Carlo, em mil R\$.

\begin{tabular}{|c|c|c|c|c|c|c|c|c|c|c|}
\hline Nome & ROI & $\begin{array}{c}\mathbf{N}^{\circ} \mathrm{DE} \\
\text { CLIENTES }\end{array}$ & $\begin{array}{c}\text { FAT/ } / \\
\text { CLIENTE }\end{array}$ & $\begin{array}{l}\text { VDAS } \\
\text { / CST/ } \\
\text { CLIENTE }\end{array}$ & CIRCUL & $\begin{array}{c}\text { DESP } \\
\text { COM/ } \\
\text { CLIENTE }\end{array}$ & $\begin{array}{l}\text { INV / } \\
\text { PERM }\end{array}$ & $\begin{array}{l}\text { DESP } \\
\text { ADM }\end{array}$ & $\begin{array}{l}\text { PASS } \\
\text { FUNC }\end{array}$ & $\begin{array}{c}\text { OUTRAS } \\
\text { DESP }\end{array}$ \\
\hline 792 & $-13,29$ & 334 & 29.228 & 18.516 & 13.167 & 5.012 & 11.032 & 2.397 & 10.434 & 515 \\
\hline 3111 & $-13,89$ & 329 & 28.795 & 19.054 & 13.575 & 5.768 & 11.029 & 2.104 & 8.791 & 601 \\
\hline 12036 & $-15,45$ & 349 & 30.942 & 17.837 & 13.173 & 6.227 & 11.096 & 2.766 & 12.402 & 561 \\
\hline 13103 & $-14,84$ & 308 & 28.766 & 20.927 & 14.250 & 5.498 & 12.621 & 2.045 & 9.061 & 573 \\
\hline 23298 & $-12,87$ & 335 & 31.010 & 18.560 & 12.915 & 6.746 & 10.103 & 2.404 & 8.537 & 497 \\
\hline 27944 & $-13,33$ & 319 & 31.046 & 19.418 & 13.166 & 5.443 & 11.716 & 2.760 & 9.743 & 396 \\
\hline 31879 & $-14,42$ & 337 & 29.517 & 19.759 & 12.764 & 5.394 & 12.610 & 2.647 & 8.514 & 419 \\
\hline 39998 & $-13,73$ & 332 & 28.997 & 20.358 & 13.312 & 5.141 & 10.977 & 2.053 & 9.427 & 339 \\
\hline 40391 & $-16,39$ & 355 & 30.865 & 19.339 & 13.123 & 6.360 & 11.044 & 2.491 & 11.574 & 486 \\
\hline 44068 & $-13,03$ & 346 & 26.672 & 17.352 & 12.817 & 4.834 & 11.826 & 2.178 & 9.562 & 562 \\
\hline 47788 & $-14,30$ & 360 & 30.233 & 20.591 & 12.759 & 4.666 & 10.323 & 2.186 & 10.647 & 468 \\
\hline 52352 & $-13,23$ & 302 & 29.958 & 18.745 & 11.807 & 5.586 & 12.356 & 2.467 & 9.386 & 425 \\
\hline 54954 & $-12,87$ & 365 & 30.987 & 18.697 & 13.556 & 6.300 & 12.045 & 2.875 & 9.311 & 455 \\
\hline 61911 & $-13,02$ & 390 & 28.498 & 17.858 & 13.086 & 5.451 & 9.901 & 2.437 & 8.906 & 485 \\
\hline 62716 & $-14,03$ & 356 & 29.258 & 19.072 & 11.818 & 6.447 & 9.430 & 1.741 & 9.249 & 395 \\
\hline 72963 & $-14,44$ & 347 & 29.222 & 19.040 & 13.455 & 5.757 & 10.782 & 2.460 & 9.201 & 393 \\
\hline
\end{tabular}




\begin{tabular}{l|c|c|c|c|c|c|c|c|c|c}
\hline Nome & ROI & $\begin{array}{c}\mathbf{N}^{\circ} \text { DE } \\
\text { CLIENTES }\end{array}$ & $\begin{array}{c}\text { FAT/ } \\
\text { CLIENTE }\end{array}$ & $\begin{array}{c}\text { VDAS } \\
\text { / CST/ } \\
\text { CLIENTE }\end{array}$ & CIRCUL & $\begin{array}{c}\text { DESP } \\
\text { COM/ } \\
\text { CLIENTE }\end{array}$ & $\begin{array}{c}\text { INV / } \\
\text { PERM }\end{array}$ & $\begin{array}{c}\text { DESP } \\
\text { ADM }\end{array}$ & $\begin{array}{c}\text { PASS } \\
\text { FUNC }\end{array}$ & $\begin{array}{c}\text { OUTRAS } \\
\text { DESP }\end{array}$ \\
\hline $\mathbf{7 4 1 7 0}$ & $-13,35$ & 376 & 29.240 & 18.267 & 13.228 & 6.662 & 13.508 & 2.550 & 9.431 & 457 \\
$\mathbf{7 4 4 3 7}$ & $-13,83$ & 320 & 29.708 & 18.097 & 12.991 & 6.664 & 10.045 & 2.422 & 9.104 & 288 \\
$\mathbf{9 0 4 8 6}$ & $-14,42$ & 375 & 29.777 & 20.257 & 13.726 & 6.391 & 11.492 & 2.392 & 7.651 & 374 \\
$\mathbf{9 3 6 9 0}$ & $-17,63$ & 375 & 30.034 & 19.898 & 12.888 & 6.681 & 10.862 & 2.554 & 8.931 & 405 \\
\hline
\end{tabular}

Fonte: Elaboração própria

Pela Tabela 5, fica constatado o comportamento das variáveis de Custo, Despesas Administrativas e Despesas Comerciais. O input Custo, que teve média de \$15.662,00 mil apurados através das médias dos balancetes, agora apresentou média de $\$ 19.101,00$ mil por meio dos resultados simulados.

Já para o input Despesas Comerciais, foi apurado o valor de $\$ 1.888,00$ mil por meio das médias dos balancetes. No entanto, se considerar os 20 piores resultados simulados, este valor apresenta a média $\$ 5.879,00$ mil. Comportamento semelhante também para o input Despesas Administrativa, cujo valor apurado por meio dos balancetes apresentou média de \$1.968; já nos resultados dos 20 piores resultados da simulação, esta média ficou em \$2.406,00 mil.

Vale ressaltar também que, mesmo nesses cenários negativos, o comportamento das variáveis de receita, que apresenta correlação positiva com o ROI, não apresenta grandes variações. É o caso do input Vendas/Faturamento, que nos valores obtidos através dos balancetes apresentou média de \$31.012,00 mil. Já a média dos 20 menores resultados estimados para o ROI ficou em \$29.722,00 mil, ou seja, uma variação pouco expressiva quando observado os altos valores negativos do ROI.

Mais uma vez, estes dados reforçam a necessidade de uma gestão que tenha como prioridade o foco nas contas de custos e despesas devido a sua grande correlação com a variável de interesse.

\section{CONCLUSÃO}

Esta pesquisa se desenvolveu no sentido de oferecer aos gestores uma ferramenta mais adequada para avaliar as informações contábeis que eles já tinham, tornando assim o processo de tomada de decisão mais completo e eficiente. Os métodos escolhidos foram a Análise de Sensibilidade e Simulação de Monte Carlo, sendo ambos amparados por Bonizio (2005).

Ao aplicarmos este teste na empresa selecionada, com dados referentes ao ano de 2015, foi possível ampliar o horizonte de ação e decisão dos gestores. O conhecimento sobre o comportamento dos inputs em cenários futuros permite a implementação de novas políticas de gestão, com focos específicos de acordo os resultados atingidos.

Ressalta-se ainda que a pesquisa possui algumas limitações, sendo a principal delas quanto à qualidade da informação previamente gerada. A classificação contábil dos lançamentos deve seguir um adequado padrão de contabilidade e estar de acordo com as Normas Brasileiras de Contabilidade (NBC). Por não ser objeto deste estudo, tal situação não foi verificada.

Como sugestão para a continuidade deste trabalho, temos que este método aqui utilizado poderia ser adotado em outras áreas da empresa e ser aplicado a outros indicadores. Como são diversos os indicadores contábeis e financeiros, temos uma série de dados que podem ser considerados inputs para outros outputs, e dessa maneira servir de base para novos testes de simulação e sensibilidade.

Portanto, a pesquisa conclui-se de forma satisfatória, pois atingiu seu objetivo. Após os testes realizados, foi possível observar e mensurar em uma base confiável como é o comportamento de cada input selecionado. Os resultados obtidos após a simulação de Monte Carlo foram suficientes para oferecer ao gestor uma nova perspectiva sobre os indicadores analisados afetando, assim, positivamente a decisão tomada por eles.

\section{REFERÊNCIAS}

ASSAF NETO, A. Finanças Corporativas e Valor. 7a. ed. São Paulo: Atlas, 2014. 824p.

BALL, Ray; BROWN, Philip. An empirical evaluation of accounting income numbers. Journal of accounting research, p. 159178,1968

BONIZIO, R. C. Análise da sensibilidade do valor econômico agregado: um estudo aplicado nas empresas de capital aberto no Brasil. São Paulo: USP, 2005. 117p. Tese (Doutorado em Controladoria e Contabilidade) - Programa de Pós-Graduação em Controladoria e Contabilidade, Faculdade de Economia, Administração e Contabilidade, Universidade de São Paulo, São Paulo, 2005.

GIL, A. C. Como Elaborar Projetos de Pesquisas. São Paulo: Atlas, 2002. 184p.

GUJARATI, D. N. Econometria Básica. $3^{\text {a }}$ ed. São Paulo: Makron Books, 2005. 860p.

ICHSANI, Sakina; SUHARDI, Agatha Rinta. The effect of return on equity (ROE) and return on investment (ROI) on trading volume. Procedia-Social and Behavioral Sciences, v. 211, p. 896-902, 2015. 
JACOBSON, Robert et al. The validity of ROI as a measure of business performance. American Economic Review, v. 77, n. 3, p. 470-478, 1987.

KANG, Moonsoo; KHAKSARI, Shahriar; NAM, Kiseok. Corporate investment, short-term return reversal, and stock liquidity. Journal of Financial Markets, v. 39, p. 68-83, 2018.

KRISTJANSDOTTIR, Katrin et al. Return on investment from the use of product configuration systems-A case study. Computers in Industry, v. 100, p. 57-69, 2018.

MENEZES, Mozart BC; KIM, Seokjin; HUANG, Rongbing. Return-on-investment (ROI) criteria for network design. European Journal of Operational Research, v. 245, n. 1, p. 100-108, 2015.

PHILLIPS, P. C. B.; SUL; D. Bias in dynamic panel estimation with fixed effects, incidental trends and cross section dependence. Journal of Econometrics, v. 137, p. 162-188, 2007.

SALTELLI, A. Sensitivity analysis for importance assessment. Risk Analysis, v. 22, n. 3, p. 579-590, 2002.

SAMSON, D. Managerial Decision Analysis. Homewood: Irwin, 1988. 720p.

SCOTT, W. R. Financial Accounting Theory. 7a. ed. London: Pearson, 2015. 571p.

WATSON, H. J.; BLACKSTONE JUNIOR., J. H. Computer Simulation. 2a. ed. New York: John Wiley \& Sons, 1989. 608p.

WILLIAMSON, O. E. Strategizing, economizing, and economic organization. Strategic Management Journal, v. 12, n. S2, p. 75-94, 1991 